\title{
Macitentan: entry-into-humans study with a new endothelin receptor antagonist
}

\author{
Patricia N. Sidharta • Paul L. M. van Giersbergen • \\ Atef Halabi • Jasper Dingemanse
}

Received: 30 November 2010 / Accepted: 23 March 2011 /Published online: 4 May 2011

(C) The Author(s) 2011. This article is published with open access at Springerlink.com

\begin{abstract}
Purpose To study the pharmacokinetics, pharmacodynamics, and tolerability of rising single doses of macitentan, an endothelin receptor antagonist, in healthy male subjects. Methods This double-blind, placebo-controlled study was performed in seven groups of eight healthy male subjects. Doses of $0.2,1,5,25,100,300$ and $600 \mathrm{mg}$ or placebo (two subjects per group) were administered. Plasma macitentan and endothelin-1 and serum total bile salt concentrations were measured and analysed non-compartmentally. Plasma and urine were analysed qualitatively for the presence of metabolites and one of these, ACT-132577, was also measured quantitatively in plasma. Standard tolerability measurements were performed throughout the study.

Results Macitentan was slowly absorbed and, at a dose of $300 \mathrm{mg}$, the $\mathrm{t}_{1 / 2}$ (95\% confidence interval, CI) was $17.5 \mathrm{~h}$ $(14.1,21.8)$. The dose-proportionality coefficient $\beta$ for $\mathrm{C}_{\max }(95 \% \mathrm{CI})$ was $0.83(0.79,0.87)$ indicating less than dose-proportional pharmacokinetics of macitentan. In plasma, a pharmacologically active oxidative depropyl metabolite, ACT-132577, was found whereas in urine two minor metabolites were detected. The $\mathrm{t}_{1 / 2}$ of ACT-132577 $(95 \% \mathrm{CI})$ was $65.6 \mathrm{~h}(53.1,80.9)$. Macitentan dose-dependently increased endothelin- 1 concentrations up to 2.2 -fold $(95 \%$ CI $1.4,2.4)$ at a dose of $600 \mathrm{mg}$, but had no consistent effect on total bile
\end{abstract}

P. N. Sidharta $(\varangle) \cdot$ P. L. M. van Giersbergen · J. Dingemanse Department of Clinical Pharmacology,

Actelion Pharmaceuticals Ltd,

Gewerbestrasse 16,

4123 Allschwil, Switzerland

e-mail: patricia.sidharta@actelion.com

\section{A. Halabi}

Clinical Research Services Kiel GmbH,

Lornsenstrasse 7 ,

24105 Kiel, Germany salts. Macitentan was well tolerated up to and including a dose of $300 \mathrm{mg}$, the maximum tolerated dose. Headache, nausea and vomiting were dose-limiting adverse events.

Conclusion The pharmacokinetic and tolerability profile of macitentan is consistent with a once-a-day dosing regimen and warrants further investigation in clinical studies.

Keywords Endothelin receptor antagonist · Healthy subjects · Pharmacokinetics · Pharmacodynamics . Tolerability

\section{Introduction}

Endothelin-1, which is synthesised predominantly by the vascular endothelium, is one of the most potent and longestlasting vasoconstrictors known [1]. Elevated endothelin-1 levels have been found in numerous diseases, suggesting a pathophysiological role of this peptide [2]. However, to date, endothelin receptor antagonists such as bosentan have only been approved for the treatment of pulmonary arterial hypertension and systemic sclerosis and digital ulcer disease whereas for other diseases either no therapeutic benefit could be demonstrated or the benefit/risk ratio was judged to be unfavourable [2, 3]. Risks related to treatment with endothelin receptor antagonists include elevations in liver aminotransferases and oedema [4]. The effects of endothelin receptor antagonists on the liver are possibly a class effect and necessitate monitoring of liver function in patients treated with these compounds [5]. The recent withdrawal from the market of the endothelin receptor antagonist sitaxentan because of cases of unpredictable serious liver injury, illustrates the need for compounds with a reduced liver liability. Similarly, a reduced risk of oedema would constitute a major advance and would allow for the application of endothelin receptor antagonists in other 
diseases. This is demonstrated by the recent findings with darusentan in patients with treatment-resistant hypertension; while darusentan provided additional reduction in blood pressure in patients in whom hypertension could not be controlled adequately with available drugs, oedema or fluid retention occurred in $27 \%$ of the patients compared with $14 \%$ in patients treated with placebo [6].

The mechanism via which endothelin receptor antagonists induce elevations in liver aminotransferases is unknown. It has been hypothesised that inhibition of the bile salt export pump (BSEP), an ABC transporter protein mediating secretion of bile salts across the canalicular plasma membrane of hepatocytes [7], results in intracellular accumulation of bile salts and subsequent liver injury [8]. The occurrence of oedema is possibly caused by circulating endothelin- 1 via activation of the endothelin B receptor [9], suggesting that endothelin receptor antagonists that block both endothelin A and $\mathrm{B}$ receptors are less prone to causing oedema. In fact, a higher incidence of oedema was observed in patients with pulmonary arterial hypertension treated with selective endothelin receptor A antagonists, such as ambrisentan, compared with dual receptor antagonists, such as bosentan [4].

In rats, intravenous administration of bosentan leads to an acute increase in plasma bile salts (Actelion Pharmaceuticals, data on file) and this model was used to screen new compounds. Compounds were further selected based on their capacity to block both types of endothelin receptors and a high octanol/aqueous buffer partition coefficient indicative of a strong affinity for tissues. Macitentan (ACT-064992, N-[5-(4-Bromophenyl)-6-(2-(5-bromopyrimidin-2-yloxy)ethoxy)pyrimidin-4-yl]-N'-propylsulfamide) is a new compound that inhibits endothelin receptors $\mathrm{ET}_{\mathrm{A}}$ and $\mathrm{ET}_{\mathrm{B}}$, but does not increase circulating bile salts in rats (Actelion Pharmaceuticals, data on file). The structure of macitentan is shown in Fig. 1. Macitentan is a lipophilic compound with a volume of distribution of $11 / \mathrm{kg}$ and a halflife of $2 \mathrm{~h}$ in rats. One circulating metabolite was identified, ACT-132577, formed by oxidative depropylation of macitentan, which has a similar volume of distribution, but a longer half-life ( $8.5 \mathrm{~h})$ [10]. It has been hypothesised that by targeting endothelin receptors in tissues macitentan could constitute an improved treatment of pulmonary arterial hypertension or other diseases in which the tissue endothelin system is activated [10]. The current entry-into-humans study was performed to investigate the pharmacokinetics, pharmacodynamics and tolerability of ascending single doses of this new compound in healthy male subjects.

\section{Materials and methods}

The study followed the principles of the Declaration of Helsinki and Good Clinical Practice and the protocol was approved by<smiles>CCCNS(=O)(=O)Nc1ncnc(OCCOc2ncc(Br)cn2)c1-c1ccc(Br)cc1</smiles>

Macitentan<smiles>NS(=O)(=O)Nc1ncnc(OCCOc2ncc(Br)cn2)c1-c1ccc(Br)cc1</smiles>

ACT-132577

Fig. 1 Chemical structure of macitentan and its main metabolite ACT-132577

an independent Ethics Committee (Ethikkommission der Ärztekammer Schleswig-Holstein, Bad Segeberg, Germany). Written informed consent was obtained from all subjects prior to study start.

Study design and subjects

This was a double-blind, randomised, placebo-controlled, single-ascending-dose study in which each dose level was investigated in a new group of eight healthy male subjects ( 6 on active drug, 2 on placebo). Doses of $0.2,1,5,25$, 100,300 and $600 \mathrm{mg}$ of macitentan were compared with placebo and the next higher dose was only administered after review of the safety and tolerability findings of subjects receiving the preceding dose. Subjects could only receive one treatment and were not allowed to re-enter another dose group. Subjects were healthy as assessed by medical history, physical examination, ECG, vital signs and clinical laboratory tests. They could not participate if they smoked, had a prior history of drug or alcohol abuse, were allergic to any drugs, were using any medication or had participated in another clinical trial during the 3-month period preceding the screening examination.

Study conduct

A screening examination was performed within 2 weeks of study drug administration. Subjects were admitted to the research unit approximately $12 \mathrm{~h}$ before study drug administration, which was performed under fasted conditions. The subjects remained in the research unit until $48 \mathrm{~h}$ 
after dosing. Later study assessments were done on an ambulatory basis. During the study days, frequent recording of vital signs and 12-lead ECG, evaluation of adverse events, blood and urine sampling for clinical laboratory testing and physical examination took place. The end-ofstudy examination took place after the 48-h (dose groups 0.2 to $100 \mathrm{mg}$ ) or $144-\mathrm{h}$ (dose groups 300 and $600 \mathrm{mg}$ ) blood sample for pharmacokinetic and pharmacodynamic purposes had been taken. An interim pharmacokinetic analysis after the 25-mg dose group had been completed revealed an unexpected long $t_{1 / 2}$ of macitentan. In order to fully characterise the elimination phase, the protocol was amended to increase the time period of follow-up after dosing from $48 \mathrm{~h}$ to $144 \mathrm{~h}$. This change in protocol was effective for the two higher dose groups only.

\section{Sampling and bioanalytics}

Plasma macitentan, its main metabolite, ACT-132577, and endothelin-1 concentrations were determined in 4-ml venous blood samples taken predose and at 0.33, 0.67, 1, 1.5, 2, 3, 4, $5,6,8,10,12,16,24,30,36,48,72,96,120$ and $144 \mathrm{~h}$ after dosing. The 72- to 144-h blood samples were only taken from subjects having received the 300 and $600 \mathrm{mg}$ doses. After collection, the tubes containing EDTA as anticoagulant were centrifuged for $10 \mathrm{~min}$ at $1,500 \mathrm{~g}$ and $4^{\circ} \mathrm{C}$. The plasma was separated and stored at $-20^{\circ} \mathrm{C}$ pending analysis.

Serum total bile salts were measured in venous blood samples taken predose and 1, 2, 3 and $4 \mathrm{~h}$ after dosing. After collection, the tubes containing no anticoagulant were left at room temperature for $30 \mathrm{~min}$ and subsequently centrifuged for $10 \mathrm{~min}$ at $1,500 \mathrm{~g}$ and $4^{\circ} \mathrm{C}$. The separated serum was stored at $-20^{\circ} \mathrm{C}$ pending analysis. No blood samples for total bile salt measurements were taken at later time points because subjects consumed a meal after the 4-h blood sample, which was expected to affect these measurements.

Prior to the start of urine collection and at the end of each collection interval, each subject was requested to completely empty his bladder. Starting at dosing, total urine over four consecutive 12-h intervals was collected in polyethylene bottles, which were kept in the refrigerator. An aliquot of $10 \mathrm{ml}$ was taken and stored at $-20^{\circ} \mathrm{C}$ until analysis.

Plasma concentrations of macitentan and its active metabolite ACT-132577 were determined using a validated liquid chromatography coupled to tandem mass spectrometry method (LC-MS/MS). The assay was linear in the concentration range $1-2,000 \mathrm{ng} / \mathrm{ml}$ and the limit of quantification was $1.0 \mathrm{ng} / \mathrm{ml}$ for both analytes. The performance of the method was monitored using quality control samples at concentrations of 3, 100 and $1,500 \mathrm{ng} / \mathrm{ml}$. At these concentrations, precision $(\% \mathrm{CV})$ was $\leq 7.1 \%$ for macitentan and $\leq 6.7 \%$ for $\mathrm{ACT}$ 132577 , whereas bias was $\leq 1.3 \%$ and $\leq 5.3 \%$ for macitentan and ACT-132577 respectively.
Plasma concentrations of endothelin-1 were determined using a commercially available radioimmunoassay ( R \& D Systems Europe Ltd, Abingdon, UK; detection limit $0.25 \mathrm{pg} / \mathrm{ml})$.

Total serum bile salts were measured using a commercially available enzymatic assay (Sigma Bile Acids Method No\#450, Trinity Biotech, Darmstadt, Germany; detection limit $1 \mu \mathrm{mol} / \mathrm{l}$ ).

\section{Metabolic profiling}

The presence of bromine atoms in the chemical structure of macitentan (Fig. 1) facilitated the detection of both parent and possible metabolites in plasma and urine. For this, $50 \mu \mathrm{l}$ of plasma from the 2-, 6- and 48-h blood samples from a subject in the 600-mg dose group were pooled and proteins precipitated with an acetonitrile/ethanol mixture $(50 / 50, \mathrm{v} / \mathrm{v})$. Similarly, $50 \mu \mathrm{l}$ of urine from each collection interval from two subjects in the 600-mg dose group were pooled. Following centrifugation of both plasma and urine at 3,360 $\mathrm{g}$ for $10 \mathrm{~min}$ at $8^{\circ} \mathrm{C}, 20 \mu \mathrm{l}$ of the extracted sample were injected onto the HPLC column (Atlantis dC18; Waters, Milford, MA, USA). Detection was performed with a LTQ Quantum mass spectrometer (ThermoFinnigan, San Jose, CA, USA) and metabolites identified with the help of the Metabolite ID software (ThermoFinnigan, San Jose, CA, USA).

\section{Data analysis}

Safety and tolerability variables were analysed descriptively. For this, subjects treated with placebo were pooled.

The pharmacokinetic variables $\mathrm{C}_{\max }, \mathrm{t}_{\max }, \mathrm{AUC}$ and $\mathrm{t}_{1 / 2}$ of macitentan and its metabolite as well as the AUC of endothelin-1 ( $\left.\mathrm{AUC}_{0-48 \mathrm{~h}}\right)$ and total bile salts $\left(\mathrm{AUC}_{0-4 \mathrm{~h}}\right)$ were determined by non-compartmental methods, as previously described [11]. Dose proportionality of macitentan pharmacokinetics was only explored by comparing logtransformed $\mathrm{C}_{\max }$ values using a power model described by Gough et al. [12], as for most dose groups no reliable estimate of $\mathrm{AUC}_{0-\infty}$ could be determined because of the unexpected long $\mathrm{t}_{1 / 2}$ of macitentan. The variables $\mathrm{AUC}_{0-48 \mathrm{~h}}$ and $\mathrm{AUC}_{0-4 \mathrm{~h}}$ for endothelin-1 and total bile salts respectively were compared between the different macitentan doses and placebo using analysis of variance (ANOVA, factor treatment). A statistically significant difference was accepted at $P<0.05$.

\section{Results}

Subjects and tolerability

Fifty-six healthy male Caucasian subjects (age range: 1949 years, body weight range: $55.4-98.0 \mathrm{~kg}$ ) participated in 
this study and all completed the study as per protocol. A summary of the adverse events reported during the study, including those adverse events judged to be unrelated to study medication, is provided in Table 1 . No unexpected adverse events, signs of oedema and/or serious adverse events were reported and all events resolved without sequelae. Up to and including a dose of $300 \mathrm{mg}$, the adverse event profile of macitentan did not significantly differ from that of placebo, whereas more adverse events than in the placebo group were reported at a dose of $600 \mathrm{mg}$. In this latter dose group, 5 out of 6 subjects reported headache of moderate intensity, accompanied by nausea and vomiting in 2 subjects. Two cases of increased alanine aminotransferase to about 2 times the upper limit of normal or less were observed: in a subject who had received $600 \mathrm{mg}$ of macitentan and the other in a subject who had received placebo. Both cases occurred 7 days after drug administration, whereas $48 \mathrm{~h}$ after administration liver enzymes were normal. In neither subject was a significant increase in aspartate aminotransferase, bilirubin or alkaline phosphatase noted, nor were there any changes in total bile salt concentrations. There were no clinically significant drug-related effects on vital signs, ECG and clinical laboratory variables.

\section{Pharmacokinetics}

The mean plasma concentration-time profiles and the pharmacokinetic variables of macitentan and its main metabolite are shown in Fig. 2, and Tables 2 and 3. Under fasting conditions, macitentan was absorbed slowly with a median $\mathrm{t}_{\max }$ varying from 8 to $30 \mathrm{~h}$ for the different dose groups. After attainment of $\mathrm{C}_{\max }$, plasma concentrations decreased slowly. The apparent terminal half-life could only be reliably estimated for the two higher dose groups and was about $16 \mathrm{~h}$. The pharmacokinetics of macitentan showed a less than dose-proportional increase for $\mathrm{C}_{\max }$ as indicated by a value for the dose proportionality coefficient $\beta(95 \% \mathrm{CI})$ of $0.83(0.79,0.87)$. Formation of the depropyl metabolite was slow with maximum concentrations attained at least $30 \mathrm{~h}$ after dosing (Fig. 2, Table 3). In comparison to the parent compound, plasma concentrations of the metabolite were higher and elimination was slower as demonstrated by mean $\mathrm{t}_{1 / 2}$ values varying from 40.2 to $65.6 \mathrm{~h}$. This resulted in a total exposure $\left(\mathrm{AUC}_{0-\infty}\right)$ to the metabolite that was 2.7 -fold $(95 \%$ CI $2.5,2.9)$ greater than that to macitentan at a dose of $600 \mathrm{mg}$.

One subject in the 600-mg dose group was excluded from the pharmacokinetic analysis because of an atypical plasma concentration-time profile of both macitentan and its metabolite. The $\mathrm{C}_{\max }$ values for parent and metabolite were within the range observed for the other subjects, but the elimination of both compounds was much slower.

\section{Metabolic profiling}

The investigation of the metabolic profile showed that in plasma only two compounds could be identified containing bromine atoms, macitentan and its metabolite, ACT132577. In urine, the presence of two other compounds was shown, one that corresponds to a metabolite formed by hydrolysis of either macitentan or ACT-132577, and the other compound, based on the molecular weight, corresponds to a glucoside analogue of ACT-132577. Because in urine no macitentan and ACT-132577 could be detected, the collected urine samples were not analysed for these compounds.

\section{Pharmacodynamics}

Plasma endothelin-1 concentrations dose-dependently increased following administration of macitentan (Fig. 3) and this effect was statistically significant for doses of $25 \mathrm{mg}$ and
Table 1 Overview of reported adverse events by treatment

Number of subjects reporting adverse events after having received macitentan ( $n=6$ per dose) or placebo $(n=14)$

Adverse events reported more than once by the same subject were counted once

\begin{tabular}{lllllllll}
\hline Adverse event & \multicolumn{7}{l}{ Treatment (mg macitentan) } & \\
\cline { 2 - 8 } & 0.2 & 1 & 5 & 25 & 100 & 300 & 600 & Placebo \\
\cline { 2 - 8 } & 0 & 0 & 0 & 2 & 1 & 2 & 5 & 3 \\
Headache & 0 & 0 & 0 & 0 & 0 & 1 & 0 & 1 \\
Back pain & 0 & 0 & 0 & 0 & 0 & 0 & 1 & 1 \\
Hepatic function abnormal & 0 & 0 & 0 & 0 & 0 & 0 & 2 & 0 \\
Nausea & 0 & 0 & 0 & 0 & 0 & 0 & 2 & 0 \\
Rhinitis & 0 & 0 & 0 & 0 & 0 & 0 & 2 & 0 \\
Vomiting & 0 & 0 & 0 & 0 & 0 & 0 & 1 & 0 \\
Abdominal pain & 0 & 0 & 0 & 0 & 0 & 0 & 1 & 0 \\
Flushing & 0 & 0 & 0 & 0 & 1 & 0 & 0 & 0 \\
Leukocytosis & 0 & 0 & 0 & 1 & 0 & 0 & 0 & 0 \\
Nasopharyngitis & 0 & 0 & 0 & 0 & 1 & 0 & 0 & 0 \\
Neck pain & & & & & & & \\
\hline
\end{tabular}



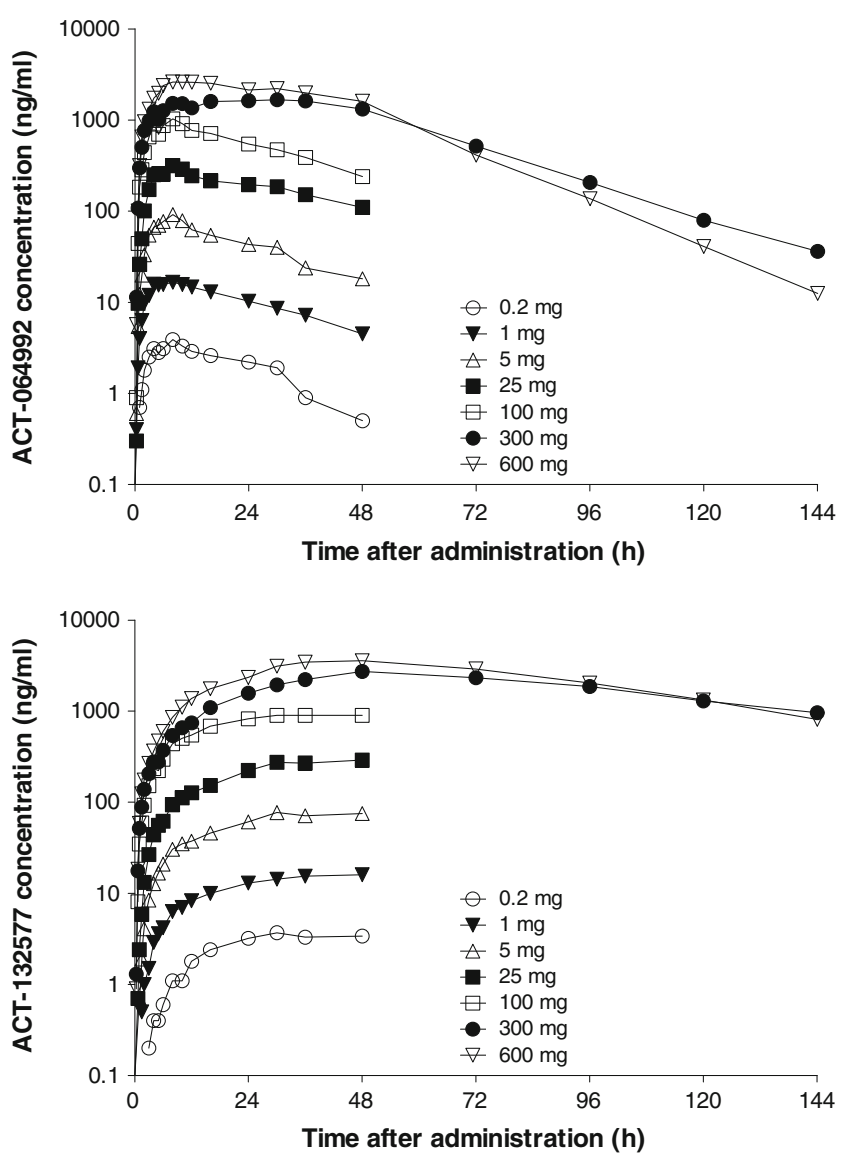

Fig. 2 Arithmetic mean plasma concentration-time profiles of macitentan (top panel) and ACT-132577 (bottom panel) in healthy subjects ( $n=6$ per group except for $600 \mathrm{mg}$ dose where $n=5$ ) after administration of a single dose of $0.2,1,5,25,100,300 \mathrm{or} 600 \mathrm{mg}$ of macitentan

higher. The maximum effect was observed with the dose of $600 \mathrm{mg}$, which increased endothelin-1 concentrations 2.2-fold ( $95 \%$ CI 1.4, 3.4) compared with placebo. Administration of macitentan had no dose-dependent effect on serum total bile salt concentration at single doses of up to $600 \mathrm{mg}$, although a small but statistically significant effect $(P<0.05)$ was observed for the lowest dose (Fig. 3).

\section{Discussion}

This study has shown that single-dose administration of macitentan in doses of up to and including $300 \mathrm{mg}$ was well tolerated in healthy male subjects. The dose-limiting adverse events of headaches sometimes accompanied by nausea and vomiting are well known for endothelin receptor antagonists [13-15]. The maximum tolerated dose of $300 \mathrm{mg}$ is thought to be well above a clinically effective dose, as based on endothelin-1 levels (see below). No treatment-related effects on any safety assessment, including liver function tests, were observed in this study. Two cases of elevated alanine aminotransferase were detected in this study. One subject was treated with $600 \mathrm{mg}$ macitentan, while the other subject was treated with placebo. It is not uncommon to see elevations in liver aminotransferases in phase I studies. In a pooled population from 13 phase I studies in which a total of 93 healthy subjects were administered placebo, $7.5 \%$ had at least one value of alanine aminotransferase that was twice the upper limit of normal [16]. Because of the small sample size in this study, the clinical relevance of these findings and their relationship to drug exposure is unknown, and has to be further evaluated in subsequent clinical trials.

The risk of liver enzyme elevations in patients treated with endothelin receptor antagonists may be a class effect and necessitates monitoring of liver function. Recently, the endothelin receptor antagonist sitaxentan was withdrawn from the market because of unpredictable serious liver injury. Preclinical models have demonstrated that some endothelin receptor antagonists, including sitaxentan, are substrates of and inhibit human hepatic transporters, suggesting a mechanism for the increased hepatotoxicity

Table 2 Pharmacokinetic variables of macitentan in healthy subjects after administration of a single dose of 0.2, 1, 5, 25, 100,300 and $600 \mathrm{mg}$ of macitentan

\begin{tabular}{lllllll}
\hline Dose $(\mathrm{mg})$ & $n$ & $\mathrm{C}_{\max }(\mathrm{ng} / \mathrm{ml})$ & $\mathrm{t}_{\max }(\mathrm{h})$ & $\mathrm{AUC}_{0-48}$ (ng.h/ml) & $\mathrm{AUC}_{0-\infty}(\mathrm{ng} . \mathrm{h} / \mathrm{ml})$ & $\mathrm{t}$ \\
\hline 0.2 & 6 & $4.0(2.6$ to 6.2$)$ & $8(8-12)$ & $85.9(52.4$ to 141$)$ & $\mathrm{ND}$ & $\mathrm{ND}$ \\
1 & 6 & $17.9(12.4$ to 25.9$)$ & $8(4-10)$ & $439(271$ to 711$)$ & $\mathrm{ND}$ & $\mathrm{ND}$ \\
5 & 6 & $93.4(79.1$ to 110$)$ & $8(4-8)$ & $2056(1,855$ to 2,278$)$ & $\mathrm{ND}$ & $\mathrm{ND}$ \\
25 & 6 & $335(264$ to 425$)$ & $8(4-30)$ & $8,810(7,412$ to 10,472$)$ & $\mathrm{ND}$ & $\mathrm{ND}$ \\
100 & 6 & $999(643$ to 1552$)$ & $8(4-12)$ & $25,281(18,775$ to 34,040$)$ & $\mathrm{ND}$ & $\mathrm{ND}$ \\
300 & 6 & $1,847(1,409$ to 2,422$)$ & $30(10-48)$ & $67,109(48,751$ to 92,380$)$ & $103,007(76,650$ to 138,428$)$ & $17.5(14.1$ to 21.8$)$ \\
600 & $5^{*}$ & $2,967(2,233$ to 3,943$)$ & $12(8-30)$ & $96,530(70,006$ to 133,102$)$ & $127,104(82,657$ to 195,450$)$ & $13.4(11.3$ to 15.9$)$ \\
\hline
\end{tabular}

Data are expressed as geometric means (and $95 \% \mathrm{CI}$ ) or for $\mathrm{t}_{\max }$ the median (and range)

$\mathrm{ND}=$ not determined because $\mathrm{t}_{1 / 2}$ could not be reliably assessed

*One subject was excluded from the descriptive statistics because of an atypical plasma concentration-time profile 
Table 3 Pharmacokinetic variables of ACT-132577 in healthy subjects after administration of a single dose of $0.2,1,5,25,100,300$ and $600 \mathrm{mg}$ of macitentan

\begin{tabular}{lllllll}
\hline Dose (mg) & $n$ & $\mathrm{C}_{\max }(\mathrm{ng} / \mathrm{ml})$ & $\mathrm{t}_{\max }(\mathrm{h})$ & $\mathrm{AUC}_{0-48}$ (ng.h/ml) & $\mathrm{AUC}_{0-\infty}$ (ng.h/ml) & $\mathrm{t}$ \\
\hline 0.2 & 6 & $3.7(2.6$ to 5.2$)$ & $36(30-48)$ & $114(77.8$ to 168$)$ & $\mathrm{ND}$ & $\mathrm{ND}$ \\
1 & 6 & $16.3(12.6$ to 21.2$)$ & $48(30-48)$ & $527(415$ to 670$)$ & $\mathrm{ND}$ & $\mathrm{ND}$ \\
5 & 6 & $84.1(74.2$ to 95.4$)$ & $33(30-48)$ & $2,540(2,117$ to 3,048$)$ & $\mathrm{ND}$ & $\mathrm{ND}$ \\
25 & 6 & $304(271$ to 342$)$ & $42(30-48)$ & $9,146(7,297$ to 11,463$)$ & $\mathrm{ND}$ & $\mathrm{ND}$ \\
100 & 6 & $931(674$ to 1,287$)$ & $42(30-48)$ & $32,068(22,727$ to 45,249$)$ & $\mathrm{ND}$ & $65.6(53.1,80.9)$ \\
300 & 6 & $2,585(1,759$ to 3,798$)$ & $48(48-72)$ & $67,174(45,343$ to 99,515$)$ & $330,549(257,489$ to 424,340$)$ & 6 \\
600 & $5^{*}$ & $3,688(2,591$ to 5,249$)$ & $48(36-48)$ & $104,968(73,339$ to 150,238$)$ & $342,084(213,414$ to 548,331$)$ & $40.2(34.6,46.7)$ \\
\hline
\end{tabular}

Data are expressed as geometric means (and $95 \% \mathrm{CI}$ ) or for $\mathrm{t}_{\max }$ the median (and range)

$\mathrm{ND}=$ not determined because $\mathrm{t}_{1 / 2}$ could not be reliably assessed

* One subject was excluded from the descriptive statistics because of an atypical plasma concentration-time profile

observed with these agents [17]. In these models macitentan did not inhibit human hepatic transporters (Actelion Pharmaceuticals, data on file), suggesting that macitentan, unlike other endothelin receptor antagonists, may not
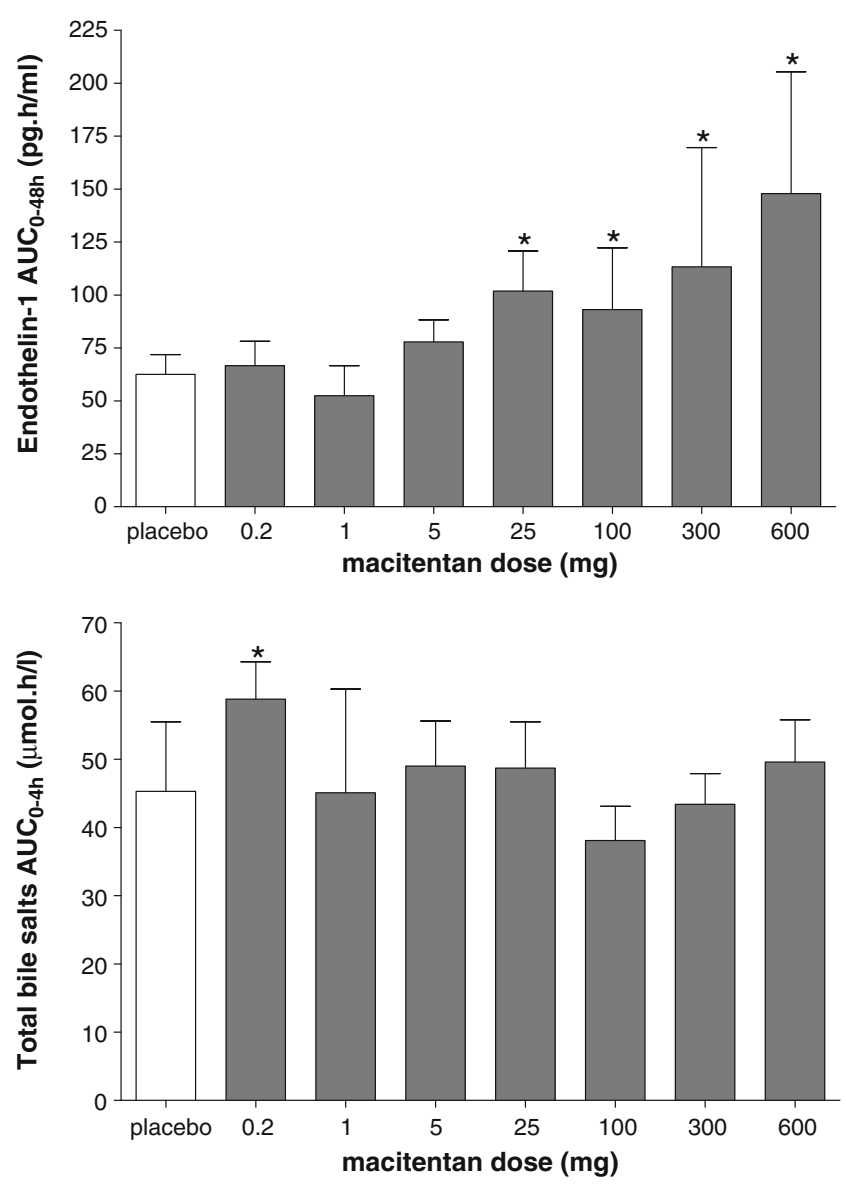

Fig. 3 Arithmetic mean $( \pm \mathrm{SD})$ plasma endothelin-1 (top panel) and serum total bile salt (bottom panel) concentrations in healthy subjects after administration of a single dose of $0.2,1,5,25,100,300$ or $600 \mathrm{mg}$ of macitentan ( $n=6$ per dose group) or placebo $(n=14)$. $* P<0.05$ compared with placebo induce cholestasis. In this study, serum total bile salt concentrations were studied during a 4-h observation period. During this time, administration of macitentan had no effect on serum total bile salt concentrations, providing some support that, in contrast to other endothelin receptor antagonists, macitentan may be devoid of liver toxic effects in man. It must be noted that both macitentan and ACT132577 have a long half-life and $t_{\max }$ occurring after the end of the 4-h observation period and that macitentan was administered as a single dose only. As elevations in liver aminotransferases induced by endothelin receptor antagonists are typically seen after repeated dosing and sometimes only after months of treatment [18], trials with longer duration of treatment are needed to truly demonstrate a lack of liver injury associated with macitentan.

The pharmacokinetics of macitentan were characterised by slow absorption and elimination. The $t_{\max }$ in most subjects was within the range 4 to $12 \mathrm{~h}$, but was up to $48 \mathrm{~h}$ in some. It is probably a chance finding that these latter subjects were part of the 300-mg dose group leading to a median $\mathrm{t}_{\max }$ of $30 \mathrm{~h}$, whereas it was 8 to $12 \mathrm{~h}$ in the other dose groups. The $t_{1 / 2}$ of macitentan and of its main metabolite indicates that a once-a-day dosing regimen will be appropriate for future studies. Such a regimen would provide increased convenience for patients compared with bosentan, which is dosed twice daily. The long $t_{1 / 2}$ of especially the depropyl metabolite suggests that accumulation will occur upon repeated dosing with macitentan. Accumulation factors of 1.7 and 10 are predicted for macitentan and its metabolite respectively based on simulation of multiple-dose profiles using the present single-dose data (data not shown). This, together with the observed higher exposure to the metabolite compared with the parent compound after single-dose administration indicates that the metabolite will contribute to a significant extent to the pharmacological activity of macitentan. It should be noted, however, that ACT-132577 is a less potent 
endothelin receptor antagonist than macitentan in both receptor binding and functional assays [10].

The pharmacokinetics of macitentan were less than dose proportional over the wide dose range investigated. However, dose-normalized $\mathrm{C}_{\max }$ values were similar for doses up to $5 \mathrm{mg}$, but decreased at higher doses (data not shown). It is expected that the small deviation from dose proportionality will not be of clinical relevance, but this remains to be demonstrated.

For an as yet unknown reason, one subject eliminated macitentan and its depropyl metabolite only very slowly. Of note, the reported case of elevated alanine aminotransferase in the 600-mg dose group did not occur in this subject. Administration of $600 \mathrm{mg}$ of macitentan was well tolerated by this subject who did not report adverse events or abnormal findings in any of the other safety assessments. Further, review of the pharmacodynamic data of this subject did not indicate relevant differences compared with the other subjects treated with the same dose. Due to the small sample size of six subjects per dose, it is not clear whether this is an isolated finding, a sample handling error, or whether this reflects a population heterogeneity in clearance of macitentan and ACT-132577. The collection of pharmacokinetic data in further studies will help to investigate this further. In current clinical development the highest dose used in multiple-dose treatment regimens is $10 \mathrm{mg}$ of macitentan once daily. With over 150 healthy subjects tested in the phase I program, no further cases of atypical plasma concentration-time profiles have been observed. Therefore, it is unlikely that genetic polymorphism in drug disposition is associated with clinically relevant differences in pharmacokinetics of macitentan and ACT-132577 in a therapeutic dose range.

The metabolic profiling experiments indicated the presence of one major depropyl metabolite, ACT-132577, in plasma and of two minor metabolites in urine and the absence of the parent compound and ACT-132577 in urine. It should be noted that the sensitivity of the method used to detect possible metabolites is limited, which may explain why metabolites were found in urine but not in plasma. Further, less abundant metabolites may have been missed, as well as those without bromine atoms. This type of metabolic profiling may be useful in early clinical development in order to quickly obtain information on metabolism, but it cannot replace a dedicated study with a radiolabelled compound. Preclinical experiments have shown the presence of ACT-132577 in rat in which it was the only circulating metabolite, and that it has affinity for $\mathrm{ET}_{\mathrm{A}}$ and $\mathrm{ET}_{\mathrm{B}}$ receptors, although its affinity is lower than that of macitentan for these receptors. In ex vivo experiments using isolated rat aortic and tracheal preparations, it was shown that, compared with macitentan, the metabolite was up to 5-fold less potent in blocking $\mathrm{ET}_{\mathrm{A}}$ and $\mathrm{ET}_{\mathrm{B}}$ receptors [10].
Macitentan caused a dose-dependent but modest increase in the plasma concentrations of endothelin-1, similar to that of bosentan albeit with greater potency [15]. The increase in endothelin-1 by endothelin receptor antagonists is thought to be caused by blockade of $\mathrm{ET}_{\mathrm{B}}$ receptors, which are responsible for the clearance of endothelin-1 at the level of the lung [19]. Macitentan and ACT-132577 are about 50and 16-fold respectively, more selective for $\mathrm{ET}_{\mathrm{A}}$ receptors than for $\mathrm{ET}_{\mathrm{B}}$ receptors [10] and at macitentan doses that increase endothelin-1, it is thus expected that the $\mathrm{ET}_{\mathrm{A}}$ receptor will be fully blocked. Although there is an ongoing debate on the advantages and disadvantages of blocking $\mathrm{ET}_{\mathrm{B}}$ receptors in patients, there does not appear to be a major difference in clinical efficacy in treating patients with pulmonary arterial hypertension between selective $\mathrm{ET}_{\mathrm{A}}$ receptor antagonists and dual $\mathrm{ET}_{\mathrm{A}}$ and $\mathrm{ET}_{\mathrm{B}}$ receptor antagonists [20, 21]. However, a head-to-head comparison has not, as yet, been performed. From the present results it may be deduced that $25 \mathrm{mg}$ will be a clinically relevant macitentan dose, i.e. the lowest dose that significantly increased plasma endothelin-1 concentrations in this study and thus fully blocks $\mathrm{ET}_{\mathrm{A}}$ receptors. Taking the expected accumulation into account upon multiple dosing, clinically relevant doses are possibly lower.

In summary, single-dose administration of macitentan, a new endothelin receptor antagonist designed to be devoid of liver injury, was well tolerated up to doses of $300 \mathrm{mg}$ and its pharmacokinetic characteristics are consistent with once-a-day dosing. This safety and pharmacokinetic profile warrants further investigation of macitentan in clinical studies.

Acknowledgements This study was funded by Actelion Pharmaceuticals Ltd.

Open Access This article is distributed under the terms of the Creative Commons Attribution Noncommercial License which permits any noncommercial use, distribution, and reproduction in any medium, provided the original author(s) and source are credited.

\section{References}

1. Yanagisawa M, Kurihara H, Kimura S, Tomobe Y, Kobayashi M, Mitsui Y, Yazaki Y, Goto K, Masaki T (1988) A novel potent vasoconstrictor peptide produced by vascular endothelial cells. Nature 332:411-415

2. Kirkby NS, Hadoke PW, Bagnall AJ, Webb DJ (2008) The endothelin system as a therapeutic target in cardiovascular disease: great expectations or bleak house? Br J Pharmacol 153:1105-1119

3. Barton M, Yanagisawa M (2008) Endothelin: 20 years from discovery to therapy. Can J Physiol Pharmacol 86:485-498

4. Trow TK, Taichman DB (2009) Endothelin receptor blockade in the management of pulmonary arterial hypertension: selective and dual antagonism. Respir Med 103:951-962

5. Price LC, Howard LS (2008) Endothelin receptor antagonists for pulmonary arterial hypertension: rationale and place in therapy. Am J Cardiovasc Drugs 8:171-185 
6. Weber MA, Black H, Bakris G, Krum H, Linas S, Weiss R, Linseman JV, Wiens BL, Warren MS, Lindholm LH (2009) A selective endothelin-receptor antagonist to reduce blood pressure in patients with treatment-resistant hypertension: a randomised, double-blind, placebo-controlled trial. Lancet 374:1423-1431

7. Green RM, Hoda F, Ward KL (2000) Molecular cloning and characterization of the murine bile salt export pump. Gene 241:117123

8. Fattinger K, Funk C, Pantze M, Weber C, Reichen J, Stieger B, Meier PJ (2001) The endothelin antagonist bosentan inhibits the canalicular bile salt export pump: a potential mechanism for hepatic adverse reactions. Clin Pharmacol Ther 69:223-231

9. Comellas AP, Briva A, Dada LA, Butti ML, Trejo HE, Yshii C, Azzam ZS, Litvan J, Chen J, Lecuona E, Pesce LM, Yanagisawa M, Sznajder JI (2009) Endothelin-1 impairs alveolar epithelial function via endothelial ETB receptor. Am J Respir Crit Care Med 179:113-122

10. Iglarz M, Binkert C, Morrison K, Fischli W, Gatfield J, Treiber A, Weller T, Bolli MH, Boss C, Buchmann S, Capeleto B, Hess P, Qiu C, Clozel M (2008) Pharmacology of macitentan, an orally active tissue-targeting dual endothelin receptor antagonist. J Pharmacol Exp Ther 327:736-745

11. Dingemanse J, Bodin F, Weidekamm E, Kutz K, van Giersbergen $P$ (2002) Influence of food intake and formulation on the pharmacokinetics and metabolism of bosentan, a dual endothelin receptor antagonist. J Clin Pharmacol 42:283-289

12. Gough K, Hutchison M, Keene O (1995) Assessment of dose proportionality: report from the statisticians in the pharmaceutical industry/pharmacokinetics UK joint working party. Drug Inf J 29:1039-1048

13. Dingemanse J, Clozel M, van Giersbergen PLM (2002) Pharmacokinetics and pharmacodynamics of tezosentan, an intravenous dual endothelin receptor antagonist, following chronic infusion in healthy subjects. Br J Clin Pharmacol 53:355-362

14. Van Giersbergen PLM, Dingemanse J (2007) Tolerability, pharmacokinetics, and pharmacodynamics of clazosentan, a parenteral endothelin receptor antagonist. Eur J Clin Pharmacol 63:151-158

15. Weber C, Schmitt R, Birnboeck H, Hopfgartner G, van Marle SP, Peeters PAM, Jonkman JH, Jones CR (1996) Pharmacokinetics and pharmacodynamics of the endothelin-receptor antagonist bosentan in healthy human subjects. Clin Pharmacol Ther 60:124-137

16. Rosenzweig P, Miget N, Brohier S (1999) Transaminase elevation on placebo during Phase I trials: prevalence and significance. Br J Clin Pharmacol 48:19-23

17. Hartman JC, Brouwer K, Mandagere A, Melvin L, Gorczynski R (2010) Evaluation of the endothelin receptor antagonists ambrisentan, darusentan, bosentan, and sitaxsentan as substrates and inhibitors of hepatobiliary transporters in sandwichcultured human hepatocytes. Can J Physiol Pharmacol 88:682691

18. Humbert M, Segal ES, Kiely DG, Carlsen J, Schwierin B, Hoeper MM (2007) Results of European post-marketing surveillance of bosentan in pulmonary hypertension. Eur Respir J 30:338-344

19. Fukuroda T, Fujikawa T, Ozaki S, Ishikawa K, Yano M, Nishikibe $\mathrm{M}$ (1994) Clearance of circulating endothelin-1 by $\mathrm{ET}_{\mathrm{B}}$ receptors in rats. Biochem Biophys Res Commun 199:1461-1465

20. Dupuis J, Hoeper MM (2008) Endothelin receptor antagonists in pulmonary arterial hypertension. Eur Respir J 31:407-415

21. Opitz CF, Ewert R, Kirch W, Pittrow D (2008) Inhibition of endothelin receptors in the treatment of pulmonary arterial hypertension: does selectivity matter? Eur Heart J 29:19361948 